\title{
MINIMAL SURFACES WITH CONSTANT CURVATURE IN 4-DIMENSIONAL SPACE FORMS
}

\author{
Dedicated to Professor S. Sasaki on his 70 th birthday
}

\author{
KATSUEI KENMOTSU ${ }^{1}$
}

\begin{abstract}
We classify minimal surfaces with constant Gaussian curvature in a 4-dimensional space form without any global assumption. As a corollary of the main theorem, we show there is no isometric minimal immersion of a surface with constant negative Gaussian curvature into the unit 4-sphere even locally. This gives a partial answer to a problem proposed by S. T. Yau.
\end{abstract}

1. Introduction. Let $M^{m}(c)$ be a connected Riemannian $m$-manifold of constant sectional curvature $c$. In this paper we classify isometric minimal immersions of $M^{2}(K)$ into $M^{4}(c)$ without any global assumption and give some partial results for a classification of minimal immersions of $M^{2}(K)$ into $M^{5}(c)$.

Let $S^{n}(1)$ be the unit sphere in the $(n+1)$-dimensional Euclidean space $R^{n+1}$. The Clifford surface in $S^{3}(1)$ and the Veronese surface in $S^{4}(1)$ are the best known examples of minimal surfaces in space forms of positive curvature.

By a rigidity theorem of Calabi and DoCarmo and Wallach, Chern and Barbosa $[2,5,4,1]$, and a localization theorem of Wallach [10], a minimal surface with constant positive curvature in $S^{4}(1)$ is locally the totally geodesic $S^{2}(1)$ or the Veronese surface. The main theorem of this paper is

THEOREM 1. Let $x: M^{2}(K) \rightarrow M^{4}(c)$ be an isometric minimal immersion of $M^{2}(K)$ into $M^{4}(c)$. If $K=c$, then $x$ is totally geodesic. Otherwise, either

(a) $K=0, c>0$ and $x$ is a locally Clifford surface in a 3-dimensional totally geodesic submanifold $M^{3}(c)$ of $M^{4}(c)$, or

(b) $K=c / 3, c>0$ and $x$ is a locally Veronese surface in $M^{4}(c)$.

As a corollary of the theorem, we show there is no isometric minimal immersion of the hyperbolic 2-plane, $H^{2}[-1]$, into $S^{4}(1)$ even locally. This gives a partial answer to problem 101 proposed by S. T. Yau [12, p. 692].

We cannot apply the method used to prove Theorem 1 to higher codimensional cases directly. However, in $\$ 4$ we prove a nonexistence theorem for minimal

Received by the editors January 7, 1983.

1980 Mathematics Subject Classification. Primary 53C42; Secondary 53A10.

Key words and phrases. Minimal surfaces with constant curvature, minimal immersions of the hyperbolic 2-plane, higher fundamental tensors.

' Partly supported by the Grant-in-Aid for Scientific Research, The Ministry of Education, Science and Culture, Japan (1981), No. 56540004. 
immersions of $H^{2}[-1]$ into $S^{5}(1)$ under an additional condition on the 2 nd fundamental form. Thus we still do not know whether $H^{2}[-1]$ can be minimally immersed in some $S^{N}(1)(N \geqslant 5)$.

It is a pleasure to thank Professor Takashi Ogata, who carefully read the first draft of this work and found some mistakes in it, and also Professors Manfredo P. DoCarmo and Tilla Milnor for helpful criticisms on this work.

2. Preliminaries. Let $x: M^{2}(K) \rightarrow M^{5}(c)$ be an isometric minimal immersion of $M^{2}(K)$ into $M^{5}(c)$. Let $e_{1}, e_{2}, e_{3}, e_{4}, e_{5}$ be local fields of orthonormal frames in $M^{5}(c)$ such that, restricted to $M^{2}(K), e_{1}$ and $e_{2}$ are tangent to $M^{2}(K)$. Let $w_{i}$, $1 \leqslant i, j \leqslant 2$, and $w_{\alpha}, 3 \leqslant \alpha, \beta, \ldots \leqslant 5$, be the fields of dual frames of $e_{A}, 1 \leqslant$ $A, B, \ldots \leqslant 5$. The structure equations of $M^{5}(c)$ are given by $d w_{A}=\sum w_{B} \wedge w_{B A}$, $w_{A B}+w_{B A}=0$, and $d w_{A B}=\sum w_{A C} \wedge w_{C B}-c w_{A} \wedge w_{B}$. The summation is taken for repeated indices. Restricting these frames to $M^{2}(K)$, we have $d w_{12}=-K w_{1} \wedge w_{2}$ and $w_{\alpha}=0,3 \leqslant \alpha \leqslant 5$. Exterior differentiation of $w_{\alpha}$ gives $w_{i \alpha}=\sum h_{\alpha i j} w_{j}$ and $h_{\alpha i j}=h_{\alpha j i}$, where the $h_{\alpha i j}$ 's are the components of the 2 nd fundamental form of $x$. By the minimality of $x$, we find $h_{\alpha 11}+h_{\alpha 22}=0,3 \leqslant \alpha \leqslant 5$. From these formulae, the Gauss equation is represented by

$$
\sum_{\alpha}\left(h_{\alpha 11}^{2}+h_{\alpha 12}^{2}\right)=c-K \geqslant 0 .
$$

Therefore $K=c$ occurs only when the immersion is totally geodesic. Hereafter we assume $c>K$. That is, the vector valued 2 nd fundamental form $\Sigma_{\alpha}\left(\sum h_{\alpha i j} w_{i} \otimes w_{j}\right) e_{\alpha}$ does not vanish at any point of $M^{2}(K)$.

In this paper we use the theory of higher fundamental tensors developed in part 1 of [7]. Since Ogata pointed out that the proof of the $r$ th $(\geqslant 3)$ order Codazzi equation in [7] is not correct, we use only results for 2 nd fundamental tensors from [7]. However, the main theorems in [7 and 8] are valid.

We introduce some notation used in [7]. Let

$$
\begin{gathered}
K_{(2)}=\sum_{\alpha}\left(h_{\alpha 11}^{2}+h_{\alpha 12}^{2}\right), \\
N_{(2)}=\left|\sum h_{\alpha 11} e_{\alpha} \wedge \sum h_{\alpha 12} e_{\alpha}\right|^{2} .
\end{gathered}
$$

The nonnegative smooth function $N_{(2)}$ on $M^{2}(K)$ is the square of the area of the parallelogram generated by $\sum h_{\alpha 11} e_{\alpha}$ and $\sum h_{\alpha 12} e_{\alpha}$. Suppose $N_{(2)}$ is identically zero on $M^{2}(K)$. By a lemma of Otsuki [9, p. 96] (see also Lemma 2 in [7]), there exists a 3-dimensional totally geodesic submanifold $M^{3}(c)$ of $M^{5}(c)$ such that $x\left(M^{2}(K)\right)$ is contained in $M^{3}(c)$ as a minimal surface. Then we have $c>0$ and $K=0$ by Chen [3, Corollary 1].

In case $N_{(2)}$ is not identically zero, the set $\Omega_{2}=\left\{p \in M^{2}(K): N_{(2)}(p) \neq 0\right\}$ is open in $M^{2}(K)$. Since $\sum h_{\alpha 11} e_{\alpha}$ and $\sum h_{\alpha 12} e_{\alpha}$ are linearly independent at each point $x$ of $\Omega_{2}$, the 2 nd osculating space $T_{x}^{(2)}$ is spanned by those vectors and $e_{i}, 1 \leqslant i \leqslant 2$. We identify the first osculating space $T_{x}^{(1)}, x \in M^{2}(K)$, with the tangent space of $M^{2}(K)$. Let $e_{A}$ be local orthonormal frame fields such that $e_{i}, 1 \leqslant i \leqslant 2$, and $e_{\alpha}$, $3 \leqslant \alpha \leqslant 4, \operatorname{span} T_{x}^{(2)}, x \in \Omega_{2}$. Then on $\Omega_{2}$ we have

$$
W_{i 5}=0 \text {. }
$$


By taking the exterior derivative of (2.4) and using the structure equation of $M^{5}(c)$, we get $w_{i 3} \wedge w_{35}+w_{i 4} \wedge w_{45}=0$. This allows us to introduce the quantities $h_{5 i j k}$ defined by the equations

$$
\sum h_{\alpha i j} w_{\alpha 5}=\sum h_{5 i j k} w_{k} .
$$

The $h_{5 i j k}$ 's are symmetric in the Latin indices $i, j, k$ and are called components of the 3rd fundamental form of $x_{\Omega_{2}}$. By (2.5) and the minimality of $x$, we get $\sum h_{\text {siik }}=0$. Note that

$$
h_{5 i j k}=h_{5 i, k}
$$

is easily verified by the definition of the covariant derivatives of $h_{\alpha i j}$, since for $\alpha \geqslant 3$,

$$
D h_{\alpha i j}=\sum h_{\alpha i j . k} w_{k}=d h_{\alpha i j}+\sum h_{\alpha s j} w_{s i}+\sum h_{\alpha i s} w_{s j}+\sum h_{\beta i j} w_{\beta \alpha} .
$$

We set

$$
K_{(3)}=h_{5111}^{2}+h_{5112}^{2} \text {. }
$$

It is easily verified that $K_{(3)}$ is an invariant of $x$ restricted to $\Omega_{2}$ under the fixed decomposition of the normal bundle, i.e., $e_{5}$ is always considered as a normal vector orthogonal to $T_{x}^{(2)}$. We put $f_{(2)}=K_{(2)}^{2}-4 N_{(2)}$ and $H_{\alpha}=h_{\alpha 11}+i h_{\alpha 12}$. Then we have (cf. [7]) $f_{(2)}=\left|\sum H_{\alpha}^{2}\right|^{2}$ and the Codazzi equation implies

$$
\left(d \sum H_{\alpha}^{2}+4 i\left(\sum H_{\alpha}^{2}\right) w_{12}\right) \wedge\left(w_{1}-i w_{2}\right)=0 .
$$

Therefore, by Lemma 3 of [7]

$$
\Delta \log f_{(2)}=8 K
$$

wherever $f_{(2)} \neq 0$, and we get

$$
\Delta f_{(2)}=8 K f_{(2)}+\left|D f_{(2)}\right|^{2} / f_{(2)} .
$$

In general, [7] gives

$$
\frac{1}{4} \Delta K_{(2)}=-2 N_{(2)}+K K_{(2)}+K_{(3)}+\sum_{\alpha \leqslant 4}\left(h_{\alpha 11,1}^{2}+h_{\alpha 11,2}^{2}\right) .
$$

By (2.1), (2.2) and (2.12), we obtain

$$
\left|D h_{311}\right|^{2}+\left|D h_{421}\right|^{2}=2 N_{(2)}-K(c-K)-K_{(3)} .
$$

3. Proof of Theorem 1. We assume $x\left(M^{2}(K)\right)$ is contained in a totally geodesic submanifold $M^{4}(c)$ of $M^{5}(c)$. Then $K_{(3)}$ is identically zero by a lemma of Otsuki [9, p. 96]. By virtue of (2.13), we have

$$
\left|D f_{(2)}\right|^{2}=8(c-K)^{-1} f_{(2)}^{3}-16(c-2 K) f_{(2)}^{2}+8(c-K)^{2}(c-3 K) f_{(2)} .
$$

In fact, (3.1) is trivial if $f_{(2)}$ is identically zero. We assume $f_{(2)}$ is not zero. Then there exists an orthonormal frame $e_{A}$ for which $h_{\alpha i j}$ 's satisfy $h_{312}=h_{411}=0$ and $h_{311}>$ $h_{412}>0$ on an open subset of $\Omega_{2}$ (cf. Wong [11, p. 480]). With respect to these frame fields we have

$$
K_{(2)}=h_{311}^{2}+h_{421}^{2}=c-K=\text { constant; } \quad N_{(2)}=h_{311}^{2} h_{421}^{2} .
$$


Therefore we have, by (3.2) and (2.13),

$$
\begin{aligned}
\left|D N_{(2)}\right|^{2} & =4 h_{311}^{2}\left(h_{421}^{2}-h_{311}^{2}\right)^{2}\left|D h_{311}\right|^{2} \\
& =\frac{4 h_{311}^{2} h_{421}^{2}}{h_{311}^{2}+h_{421}^{2}}\left\{\left(h_{421}^{2}+h_{311}^{2}\right)^{2}-4 h_{421}^{2} h_{311}^{2}\right\}\left(2 N_{(2)}-K(c-K)\right) \\
& =4 N_{(2)}\left(K_{(2)}^{2}-4 N_{(2)}\right)\left(2 N_{(2)}-K(c-K)\right) /(c-K) .
\end{aligned}
$$

By definition of $f_{(2)}$ and (3.2), this implies (3.1).

Lemma 1. Let $x: M^{2}(K) \rightarrow M^{4}(c)$ be an isometric minimal immersion with $N_{(2)} \neq 0$ on $M^{2}(K)$. Then we have $K=c / 3$ and $c>0$. Locally $x$ is the Veronese surface in $M^{4}(c)$.

Proof. If $f_{(2)}$ is identically zero on $M^{2}(K)$, then $\sum h_{\alpha 11} e_{\alpha}$ and $\sum h_{\alpha 12} e_{\alpha}$ are orthogonal and have the same nonzero length (cf. [7, p. 475]). By normalizing these vectors, we adopt them as a part of the basis of $T_{x}^{(2)}, x \in M^{2}(K)$. For these new frame fields, we have $h_{311}=h_{412}>0, h_{312}=h_{411}=0$. The Gauss equation implies

$$
h_{311}^{2}=h_{412}^{2}=(c-K) / 2=\text { constant }>0 .
$$

We have $D h_{311}=d h_{311}=0$ and $2 w_{12}=w_{34}$, as seen by the formulas

$$
D h_{312}=h_{311}\left(2 w_{12}-w_{34}\right)=h_{311,2} w_{1}-h_{311,1} w_{2}=0 \text {. }
$$

Exterior differentiation of $2 w_{12}=w_{34}$ gives $K=h_{311} h_{421}$. It follows from these formulas that $K=h_{311}^{2}$ is positive, and we can see $K=c / 3$ by (2.13), which implies $c>0$.

It is known that such an immersion represents locally the Veronese surface (cf. for instance [11, Theorem 4.2]).

Next we will show that the case of $f_{(2)} \neq 0$ cannot happen. By (2.11) and (3.1), we obtain

$$
\Delta f_{(2)}=8(c-K)^{-1} f_{(2)}^{2}-8(2 c-5 K) f_{(2)}+8(c-K)^{2}(c-3 K) .
$$

If we set $\theta=f_{(2)}$, then (3.1) and (3.3) are expressed in the following way: $\Delta \theta=\phi(\theta)$, $|D \theta|^{2}=f(\theta)$, where $\phi(\theta)$ and $f(\theta)$ are polynomials for $\theta$ with constant coefficients. It is then proved that $\theta$ must be a constant function. If $\theta$ is not constant then there exist local coordinates $(\theta, \tau)$ on $M^{2}(K)$ such that the first fundamental form is

$$
d s^{2}=\left(d \theta^{2}+\exp \left(2 \int \phi f^{-1} d \theta\right) d \tau^{2}\right) / f(\theta)
$$

and the Gauss curvature $K$ satisfies

$$
f K+\left(\phi-f^{\prime}\right)\left(\phi-\frac{1}{2} f^{\prime}\right)+f\left(\phi^{\prime}-\frac{1}{2} f^{\prime \prime}\right)=0,
$$

where the prime denotes differentiation with respect to $\theta$ (cf. Eisenhart [6, p. 164]). The left-hand side of (3.4) is a polynomial in $\theta$ such that the coefficients of $\theta^{3}$ and $\theta$ are $8(8 c-27 K) /(c-K)$ and $8(c-K)^{2}(c-3 K)(8 c-3 K)$, respectively. Since (3.4) is a nontrivial polynomial with constant coefficients, $\theta$ must be a constant. Therefore $f_{(2)}$ is a nonzero constant, which implies $K=0$ by (2.10) and $f_{(2)}=c^{2}-$ $4 N_{(2)}$. By (3.3), we have $f_{(2)}=c^{2}$, which is a contradiction because we have assumed $N_{(2)} \neq 0$. This proves Lemma 1 . 
We prove Theorem 1 as follows: If $N_{(2)}$ is identically zero, then $x$ is totally geodesic or we have $K=0$ and $c>0$. Moreover, in this case, $x\left(M^{2}(0)\right)$ is contained in a 3-dimensional totally geodesic $M^{3}(c)$ as part of a Clifford surface [3].

When $N_{(2)} \neq 0$ and $K_{(3)} \equiv 0$, we apply the above lemma to $\Omega_{(2)}$. Since $f_{(2)}$ is identically zero on $\Omega_{(2)}, N_{(2)}$ is a positive constant on $\Omega_{(2)}$. Since $\Omega_{(2)}$ is open and closed, we have $\Omega_{(2)}=M^{2}(K)$, because $M^{2}(K)$ is connected by definition. Hence Theorem 1 has been proved.

4. The 3rd fundamental form. In this section we study the case $K_{(3)} \not 0$, which means that the minimal immersion $x: M^{2}(K) \rightarrow M^{5}(c)$ is full. We define covariant derivation for the 3 rd fundamental tensor (cf. [7]) by

$$
\tilde{D} h_{5 i j k}=\sum h_{5 i j k i l} w_{l}=d h_{5 i j k}+\sum h_{5 s j k} w_{s i}+\sum h_{5 i s k} w_{s j}+\sum h_{5 i j s} w_{s k} .
$$

Exterior differentiation of (2.5) gives

$$
\sum h_{5 i j k ; l} w_{l} \wedge w_{k}=-\sum h_{\alpha i j, l} w_{\alpha 5} \wedge w_{l} .
$$

When we set $w_{\alpha 5}=\sum a_{\alpha j} w_{j},(4.2)$ is equivalent to

$$
h_{5 i j k, l}-h_{5 i j l ; k}=\sum h_{\alpha i j, l} a_{\alpha k}-\sum h_{\alpha i j, k} a_{\alpha l} \text {. }
$$

By (2.5) we have

$$
\sum h_{\alpha i j} a_{\alpha k}=h_{5 i j k} \text {. }
$$

Lemma 2. Let $x: M^{2}(K) \rightarrow M^{5}(c)$ be a full isometric minimal immersion. Then the 3 rd order Codazzi equations

$$
h_{5 i j k: l}=h_{5 i j l: k}
$$

hold if $N_{(2)}$ is constant on $M^{2}(K)$.

Proof. $f_{(2)}$ is constant on $M^{2}(K)$. If $f_{(2)}$ is zero, then we take frame fields $e_{A}$ such that $h_{311}=h_{412}$ and $h_{312}=h_{411}=0$. In the case of $f_{(2)} \neq 0$, we take Y. C. Wong's frame, for which $h_{311}>h_{412}, h_{312}=h_{411}=0$ (cf. [11, p. 480]). Since $N_{(2)}$ is constant, in both cases, $h_{311}$ and $h_{421}$ are also locally constant, so $D h_{3 i j}=D h_{4 i j}=0$. It follows that the right-hand side of (4.2)' vanishes. Q.E.D.

Hereafter we assume $N_{(2)}$ is positive constant on $M^{2}(K) . f_{(2)}$ is also constant. Since we have $D h_{\alpha i j}=0$, by (2.13), $K_{(3)}$ is constant. We take frame fields $e_{i}$ locally for which $h_{5112}$ vanishes on a neighborhood of $M^{2}(K)$. It shall be remarked that $e_{3}$ and $e_{4}$ are any vectors such that $e_{i}, e_{3}$ and $e_{4}$ are bases of $T_{x}^{(2)}$. Since $K_{(3)}=h_{5111}^{2}+$ $h_{5112}^{2}$ is a nonzero constant, $h_{5111}$ is locally a nonzero constant. From the formulas $D h_{5111}=0$ and $D h_{5112}=3 h_{5111} w_{12}=h_{5111: 2} w_{1}-h_{5111 ; 1} w_{2}=0$, which is derived by Lemma 2, we get $w_{12}=0$. It implies $K=0$ so $c$ is positive by (2.1). Therefore Lemma 2 in [8] holds, so $x$ is locally a generalized Clifford surface of $M^{5}(c)$ described in [8]. Summarizing, we get this result.

THEOREM 2. Let $x: M^{2}(K) \rightarrow M^{5}(c)$ be a full isometric minimal immersion with $N_{(2)}$ constant on $M^{2}(K)$. Then $K=0$ and $c$ is positive. Locally $x$ is one of the generalized Clifford surfaces described in [8]. 
REMARKS. (1) If the $r$ th $(\geqslant 3)$ fundamental tensors all satisfy the $r$ th order Codazzi equation, then we can prove a local classification theorem for minimal immersions $M^{2}(K) \rightarrow M^{m}(c)$ by methods similar to those proving Theorem 1 .

(2) Let $x: M^{2}(K) \rightarrow M^{5}(c)$ be any full isometric minimal immersion. Then by (2.13) we have $\left|D h_{311}\right|^{2}+\left|D h_{421}\right|^{2} \leqslant 2 N_{(2)}-K(c-K)$. It follows that

$$
\begin{aligned}
\left|D f_{(2)}\right|^{2} & \leqslant 8(c-K)^{-1} f_{(2)}^{3}-16(c-2 K) f_{(2)}^{2}+8(c-K)^{2}(c-3 K) f_{(2)}, \\
\Delta f_{(2)} & \leqslant 8(c-K)^{-1} f_{(2)}^{2}-8(2 c-5 K) f_{(2)}+8(c-K)^{2}(c-3 K) .
\end{aligned}
$$

Under suitable assumptions on the topology of $M^{2}(K)$, the author conjectures that the $f_{(2)}$ must be constant.

\section{REFERENCES}

1. J. L. M. Barbosa, On minimal immersions of $S^{2}$ into $S^{2 m}$, Trans. Amer. Math. Soc. 210 (1975), $75-106$.

2. E. Calabi, Minimal immersions of surfaces in Euclidean spheres, J. Differential Geom. 1 (1967), 111-125.

3. B. Y. Chen, Minimal surfaces with constant Gauss curvature, Proc. Amer. Math. Soc. 34 (1972), 504-508.

4. S. S. Chern, On the minimal immersions of the two-sphere in a space of constant curvature, Problems in Analysis, Princeton Univ. Press, Princeton, N. J., 1970, pp. 27-40.

5. M. DoCarmo and N. Wallach, Minimal immersions of spheres into spheres, Ann. of Math. (2) 95 (1971), 43-62. 1947.

7. K. Kenmotsu, On compact minimal surfaces with non-negative Gaussian curvature in a space of constant curvature. I, II, Tôhoku Math. J. 25 (1973), 469-479; 27 (1975), 291-301.

8. On minimal immersions of $R^{2}$ into $S^{N}$, J. Math. Soc. Japan 28 (1976), 182-191.

9. T. Otsuki, Minimal submanifolds with m-index 2 and generalized Veronese surfaces, J. Math. Soc. Japan 24 (1972), 89-122.

10. N. Wallach, Extension of locally defined minimal immersions of spheres into spheres, Arch. Math. 21 (1970), 210-213.

11. Y. C. Wong, Contributions to the theory of surfaces in a 4-space of constant curvature. Trans. Amer. Math. Soc. 59 (1946), 467-507.

12. S. T. Yau, Seminar on differential geometry, Ann. of Math. Studies, No. 102, Princeton Univ. Press, Princeton, N. J., 1982.

Department of Mathematics, College of General Education, Tómoku University, Kawauchi, SENDAI, 980, JAPAN 